\title{
A preliminary investigation into the effects of ocular lubricants on higher order aberrations in normal and dry eve subjects
}

Samantha McGinnigle ${ }^{1}$

Frank Eperjesi ${ }^{1}$

Shehzad A Naroo ${ }^{1}$

1. School of Life and Health Sciences, Aston University, Birmingham, B4 7ET, UK

Corresponding Author: Dr Shehzad A. Naroo

Correspondence address and address for reprints: School of Life and Health Sciences, Aston University, Birmingham, B4 7ET, UK

Tel. **44 1212044132

Fax. ${ }^{* * 44} 1212044048$

EMAIL: s.a.naroo@aston.ac.uk

INTRODUCTION

Dry eye is a complex condition with an inflammatory component, characterised by changes to the ocular surface which relate to increased tear osmolarity and a reduced quantity or quality of tears. The Dry Eye Workshop (DEWS) defined dry eye as 'a multifactorial disease of the tears and ocular surface that results in symptoms of discomfort, visual disturbance, and tear film instability with potential damage to the ocular surface, accompanied by increased osmolarity of the tear film and inflammation of the ocular surface.' The key additions to their 1995 definition were the inclusion of symptoms of visual disturbance, osmolarity and inflammation $[1,2]$. The average tear film thickness has recently been estimated at $3 \mu \mathrm{m}$ [3], which if reduced, can result in symptoms of discomfort, 
changes in corneal sensitivity and a reduction of visual quality [1], where local variations in power produce higher order aberrations and degradation of the image $[4,5]$.

Changes in tear osmolarity can be measured by tests such as the TearLab system (Ocusense Inc., San Diego, CA, US), although the symptoms of discomfort can also be assessed subjectively by questionnaire or history and symptoms [1]. Sodium fluorescein is widely referred to as a 'vital dye,' although this is has been challenged as the mechanism of staining is not fully understood [6]. Standardized grading of corneal and conjunctival fluorescein staining have given this dye broad applicability as a dry-eye diagnostic test, particularly as an assessment tool in clinical studies of dry eye. Nichols reported retrospectively on a group of 447 patients with dry eye in a clinic based sample and found symptom assessment (82.8\%), fluorescein staining $(55.5 \%)$ and tear break up time $(40.7 \%)$ to be the most frequently used tests in cases with a dry eye diagnosis [7].

Symptoms of dry eye are often exacerbated by environmental conditions e.g. low humidity [8] and tasks requiring concentration, e.g. computer use $[9,10]$. The importance of symptoms is underlined by their inclusion in the definition of dry eye [1]; however, lack of correlation between signs and symptoms of dry eye limits the usefulness of data obtained in this way to assess severity [11-13]. Validated questionnaires have been used successfully as a method of screening for potential dry eye [14].

Artificial tears are the first line treatment for dry eye, but the exact mechanism of these products is difficult to identify as these preparations do not recreate the function of the tear film, but do seem to have a lubrication effect [15]. Preservatives in artificial tears have been shown to be detrimental to the ocular surface e.g. short term exposure to benzalkonium chloride has been shown to decrease goblet cell density in humans [16] and cause tear film instability [17], conjunctival squamous metaplasia and apoptosis [18], disruption of the corneal epithelium barrier [19], and have possible proinflammatory effects [20]. Unpreserved drops or alternative, less toxic preservatives are therefore preferable for the treatment of dry eye. Liposome sprays have been shown to increase lipid layer thickness and improve tear film stability in normal eyes for approximately 60 minutes following application to a closed eye [21]. The delivery system offers an advantage in that it does not require preservatives and is easy to apply. A reduction in aqueous volume has been shown to affect the spreading ability and stability of the lipid layer [22], introducing the possibility that a lubricant drop could theoretically enhance the performance of a lipid spray in dry eyes.

Historically, the use of invasive techniques to evaluate the tear film may have compromised the results, which has led to the recommendation of 'minimally invasive techniques' for the diagnosis and monitoring of dry eye [14, 23]. The use of wavefront sensing aberrometers has been shown to be suitable for evaluating the optical qualities of the tear film [24] and assessing the effects of artificial tears [25].

The purpose of this study was to examine the immediate effects of unpreserved hypromellose $0.3 \%$ $w / v$ artificial tears (Lumecare ${ }^{\circledR}$, Medicom Healthcare Ltd, Hampshire,UK) a liposome spray (Tears Again , Optima Pharmazeutische $\mathrm{GmbH}$ ) and the treatments combined, on patient-reported ocular comfort, higher order aberrations and Strehl ratio in normal and self-diagnosed dry eye subjects.

\section{METHODS}


Subjects

The study was approved by the institutional ethics committee and the research followed the tenets of the Declaration of Helsinki. The nature of the study was explained to the participants and written, informed consent was obtained. A group of 24 normals ( 12 female, 12 male) with a mean age of 24.2 (SD 8, median 21) years and dry eye subjects (15 female, 9 male) with a mean age of 25.7 (SD 7, median 22) years were recruited. The inclusion criterion for dry eye was a score of $\geq 6$ according to the Chalmers 5-item questionnaire [26]. The mean dry eye questionnaire score for the normal group was 2.7 (median 2, SD 2.3). The mean dry eye questionnaire score for the dry group was 10.7 (median 12, SD 3.2) (FIGURE 1). The exclusion criteria were: diagnosis of dry eye or any eye disease including ocular allergy, medication affecting the ocular surface, refractive surgery, contact lens wear and use of any eye drops within 24 hours prior to the study.

The measurements were conducted in a stable, air-conditioned environment of $21^{\circ}$ and $24 \%$ humidity. Subjects remained in this environment between measurements, during which time they performed tasks requiring high levels of concentration.

Methods

The subjects were assessed for all interventions administered to the right eye only on three different days within a two week period. The interventions were one drop of unpreserved hypromellose, one spray of liposome solution and the drop and spray combined. Unpreserved drops were selected as common preservatives e.g. benzalkonium chloride have a detergent effect [27] and the potential effect of this detergent on the liposome spray was unknown. Allocation of treatment order was decided for each subject using randomisation tables. Comfort levels for the right eyes were rated on a scale of 1-10, where 10 represented the most comfortable at baseline and after 1 hour. The subjects were seated with their chin on the chin-rest of the aberrometer when all lubricants were applied to enable the investigator to measure aberrations 5 seconds after intervention. Aberrometry was performed 2 seconds after a blink (aberrations are stable for up to $4 \mathrm{~s}$ after a blink[28]) at baseline, 5 seconds after treatment and 1 hour after treatment using the Nidek OPDScan III, an aberrometer/corneal topography workstation. The aberrometer works on the principle of scanning slit retinoscopy/skiascopy, measuring the time delay between the central and peripheral fundus reflexes. The difference in power across the pupil is used to generate the wavefront and autorefraction data [29] from which Zernike-based maps can be derived. The total eye wavefront error, total spherical aberration and total coma-like aberrations were recorded over a pupil diameter of $5 \mathrm{~mm}$, as this was the smallest natural pupil size in this cohort. Coma and spherical aberrations have been shown to be the most significant higher-order aberrations [30]. Magnitudes of the coefficients of Zernike polynomials were represented as the root mean square (RMS, in micrometers). The Strehl ratio for higher order aberrations was also recorded as a predictor of the image optical quality at the fovea, higher values indicating improved image quality [31]. A slit-lamp examination was performed after the final aberrometry reading to assess corneal staining using fluorescein sodium. The hypothesis for this study was that the combination of aqueous drops and a liposomal spray would result in the most stable and improved optical surface in the dry eye group after 60 minutes. The normal group was expected to exhibit minimal change after 60 minutes. 
Data analysis

The Power calculation was conducted using G*Power 3.1 [32] (ANOVA repeated measures within factor) and 24 subjects were recruited to achieve a medium effect with $80 \%$ power and an alpha level of 0.05. Statistical analysis was performed with SPSS v20.0 (SPSS INC., Chicago, USA). The ranked data was analysed using Friedman's ANOVA, with post hoc Bonferroni corrected Wilcoxon signed-rank tests. Normally distributed continuous data underwent parametric statistical analysis. Normality was confirmed for the data sets using Kolmogarov-Smirnoff. Analysis of variance (ANOVA) or 2 tailed independent t-tests were used to analyse the data. When ANOVA results were significant, post hoc Bonferroni corrected t-tests were used to control for type 1 error. A P value less than 0.05 was considered significant.

\section{RESULTS}

The comfort scores revealed the largest improvement after the combination treatment; $\chi^{2}(2)=$ $6.240 p=0.04$ (Mean improvement in normal eyes $0.7 \pm 0.2$ and dry eyes $1.4 \pm 1.1$ ), followed by spray (Mean improvement in normal eyes $0.6 \pm 0.2$ and dry eyes $1.3 \pm 1.3$ ) then drops (Mean improvement in normal eyes $0.4 \pm 0.2$ and dry eyes $1.2 \pm 1.1$ ). The scores had larger standard deviations in the dry group, although post hoc comparisons between specific interventions and eye types did not reach statistical significance and the comfort scores did not support the treatment preferences (Fig.1 and table 1)

The baseline total higher order aberrations, coma and spherical aberrations between the normal and dry groups were investigated to determine if there was a change in values across the visits before any intervention. The mean values for total higher order aberrations were slightly higher in the dry group; however, the standard deviations were large and this did not reach statistical significance on any separate occasion (Table 2 ).

A mixed ANOVA model (eye type $X$ time of measurement) was designed to determine whether the Strehl ratio for higher order aberrations showed a reduction by similar amounts in normal and dry eyes at each measurement time point. Table 3 shows the objective values and standard deviations for normal and dry eyes at baseline and 60 minutes after intervention. For the hypromellose drops there was a significant main effect for the time of measurement, $F(2,92)=9.91, p=<0.01$; Bonferroni corrected post hoc tests showed this related to the change from baseline to immediately after instillation of hypromellose drops (mean difference in normal eyes $0.130, p=\langle 0.01$; mean difference in dry eyes $0.036, p=0.01$ ), however there was no significant difference between measurements taken at baseline and an hour after intervention in either eye type. For liposome spray there was no significant effect for time of measurement, $F(2,92)=1.905, p=0.155$ or eye type, $F(1,46)=1.839, p=0.182$. For hypromellose drops and liposome spray combined there was no significant effect for time of measurement $F(2,92)=0.529, p=0.591$, or eye type $F(1,46)=$ $0.911, p=0.354$.

Instillation of hypromellose drops increased total aberrations $(F(1.36,62.61)=19.00, p<0.01$, Greenhouse-Geisser corrected) from the 'baseline' compared with 'immediately after' (mean difference $0.23, p<0.01$ for normal and dry eyes) and there was a similar sized reduction at the 
'immediately after' compared with 'hour' time points (mean difference $0.22, p<0.01$ for normal and dry eyes). There was no significant effect for eyetype, $F(1,46)=1.782, p=0.188$. Analysis for the effect of liposome spray on total aberrations showed no significant effect for time of measurement, $F(2,92)=1.756, p=0.178$ or eye type, $F(1,46)=3.060, p=0.087$. For drops and spray there was no significant effect for time of measurement $F(2,92)=4.387, p=0.15$, or eye type $F(1,46)=1.118, p$ $=0.296$.

Instillation of hypromellose drops had no significant effect on spherical aberrations for time of measurement $F(2,92)=1.282, p=0.282$ or eye type $F(1,46)=1.112, p=0.33$. The main results for time of measurement after application of liposome spray were $F(2,92)=1.112, p=0.33$, with no significant difference for eye type $F(1,46)=1.112, p=0.33$. The effect on spherical aberrations for the interventions combined was insignificant; $F(1.39,63.77)=0.836, p=0.40$, Greenhouse-Geisser corrected. There was no significant effect for eye type $(F 1,46)=0.781, p=0.38$.

Instillation of hypromellose drops had no significant effect on coma for time of measurement $\mathrm{F}$ $(1.49,63.40)=0.527, p=0.54$ or eye type $F(1,46)=0.106, p=0.74$. The main results for time of measurement after application of liposome spray were $F(2,92)=0.120, p=0.887$, with no significant difference for eye type $F(1,46)=0.086, p=0.770$. The effect on coma for the interventions combined was insignificant; $F(1.21,55.52)=1.767, p=0.189$, Greenhouse-Geisser corrected. There was no significant effect for eye type $(F 1,46)=0.071, p=0.791$.

\section{DISCUSSION}

A third of the 'dry eye' group scored 7 in the questionnaire, which only just placed them in to the 'dry' category. There is a strong possibility that the differences between the 'normal eye' and 'dry eye' groups failed to reach statistical significance due to the high proportion of borderline 'normal eyes' in the 'dry eye' group. The measurement of visual quality immediately after instillation of hypromellose drops was found to be significantly worse, in agreement with other studies investigating artificial tears [33-35], however, the non-significant result following instillation of the hypromellose drops and liposome spray combined was unexpected. This may have been due to small variations in drop size or amount of spray applied, however, the same investigator (SM) applied all interventions in an attempt to standardise the dosage. Ridder et al. measured the drop weight in their study, but concluded that it was unlikely to affect the results [33] so this is unlikely to have been a major factor. The timing of the measurements after a blink was another possible source of variation; however, the technique used followed established protocol [33]. The results are more likely to have been influenced by the psychological factor of having two interventions at the same time which may have led to increased blinking or lid squeezing, where excess volume of the drop could wash the liposome spray away. The comfort scores, however, were marginally higher for the combination of products; the subjects possibly perceiving a larger effect due to 'more' intervention, although this did not extend to the treatment preferences. The convenience of having a multi-use spray which needed no mirror to aid application was a factor commonly cited by subjects when choosing their preferred product. The non-significant post hoc tests following a significant result for the Friedman analysis of the comfort scores may indicate a Type 1 error; however, it is more likely that this reflects a lack of power, particularly in a small sample size. In this study the 60 minute time interval to question the issue of comfort may not have been long enough. Furthermore, the 
measurement of aberrometry immediately of installation of the drops and again at 60 minutes may not have been sufficient to examine the full influence of on aberrations and certainly a follow-up study with more frequent and regular measurements would be warranted.

Larger differences in higher order aberrations may have been found over an increased pupil diameter [36], however, these measurements were meant to reflect visual effects in average indoor lighting conditions. The values obtained by different methods of aberrometry have been shown to vary with respect to values for deviations in wavefronts. The automatic 'averaging' function has also been cited as a source for error as this is not an indication of reduced variance between the measurements; therefore, recommendations for multiple separate measurements have been made [37]. The differences between the dry and normal group did not reach statistical significance at the hour time point for any of interventions and there were large standard deviations in the measurements. This may be due to fluctuations in accommodation [38, 39], the variable nature of the aberrations themselves e.g. local aberrations at the border of the tear film breaking up where the slope would be steep; the complex nature of such aberrations would not be well described by Zernike modes. Acceptable tolerances for measurement with aberrometry may also mean that the error exceeds the differences in total aberrations between the normal and borderline dry eyes [36, 40].

Based on Craig's findings investigating normal eyes [21], it is not unreasonable to expect the effect in border-line dry eyes to be more short-lived and a difference to show between the groups at the 60 minute time mark. It may have been more informative to take measurements more frequently, for example every ten minutes, to see if there was a point where there was a difference between dry and normal eyes, however, the study was designed to assess the benefit of combining treatments and specifically showing a difference between normal and dry eyes at the 60 minute time point, which would have been of clinical interest.

Fluorescein sodium was used to assess corneal staining following the final aberration measurements; however, there was no staining in any participants. Ideally it would have been better to assess the cornea before treatment, but this would clearly interfere with the results due to the established destabilising effect the drug has on the tear film and the invasive nature of the test. Other studies have assessed staining on a different day to measurement for inclusion criteria [33, 34], however, due to the variable nature of the tear film, the method chosen in this study was considered to be the most effective representation of the ocular surface at the time of measurement. There is also ambiguity regarding what fluorescein staining actually represents and particularly whether it really gives a true representation of the integrity of the cornea [6].

Combining artificial tear drop and liposome spray treatments for dry eye has not been shown to improve or prolong effectivity as measured by aberrometry over a $5 \mathrm{~mm}$ pupil in dry or normal eyes. One application of any ocular lubricant gave a subjective improvement although this could not be detected by aberrometry after one hour. This may suggest that in a clinical setting, symptomatic patients with no corneal staining could benefit from ocular lubricants for symptomatic relief. 
[1] Lemp MA. The definition and classification of dry eye disease: Report of the Definition and Classification Subcommittee of the International Dry Eye WorkShop (2007). The Ocular Surface, 2007;5(2):75-92.

[2] Lemp MA. Report of the National Eye Institute/Industry workshop on clinical trials in dry eyes. Eye \& Contact Lens 1995;21(4):221.

[3] Azartash K, Kwan J,Paugh JR, Nguyen AL, Jester JV, Gratton E. Pre-corneal tear film thickness in humans measured with a novel technique.Mol Vis, 2011;17:756-67

[4] Rieger $\mathrm{G}$. The importance of the precorneal tear film for thequality of optical imaging. Br J Ophthalmol 1992;76:157-8.

[5] Koh S, Maeda N,Kuroda T, Hori Y, Watanabe H, Fujikado T, Tano Y, Hirohara Y, Mihashi T. Effect of tear film break-up on higher-order aberrations measured with wavefront sensor. American Journal of Ophthalmology 2002;134(1):115-17.

[6] Morgan PB, Maldonado-Codina C. Corneal staining: Do we really understand what we are seeing? Contact Lens and Anterior Eye, 2009;32(2):48-54.

[7] Nichols KK, Nichols JJ, Zadnik K. Frequency of dry eye diagnostic test procedures used in various modes of ophthalmic practice. Cornea, 2000;19(4):477.

[8] McCarty DJ, McCarty CA. Survey of dry eye symptoms in Australian pilots. Clinical \& Experimental Ophthalmology, 2000;28(3):169-171.

[9] Nakaishi H, Yamada Y. Abnormal tear dynamics and symptoms of eyestrain in operators of visual display terminals. Occupational and Environmental Medicine, 1999;56(1):6-9.

[10] Himebaugh NL, Begley CG, Bradley A, Wilkinson JA. Blinking and tear break-up during four visual tasks. Optometry \& Vision Science, 2009;86(2):E106-14.

[11] Johnson ME. The association between symptoms of discomfort and signs in dry eye. The Ocular Surface, 2009. 7(4): p. 199-211.

[12] Nichols, KK, Nichols JJ, Mitchell GL. The lack of association between signs and symptoms in patients with dry eye disease. Cornea, 2004;23(8):762.

[13] Vitale S,Goodman LA, Reed GF, Smith JA. Comparison of the NEI-VFQ and OSDI questionnaires in patients with Sjögren's syndrome-related dry eye. Health and Quality of Life Outcomes,

2004;2(1):44.

[14] Bron AJ, Smith JA, Calonge M, Methodologies to diagnose and monitor dry eye disease: report of the Diagnostic Methodology Subcommittee of the International Dry Eye WorkShop (2007). The Ocular Surface, 2007;5(2):108-152.

[15] Pflugfelder SC, Management and therapy of dry eye disease: report of the Management and Therapy Subcommittee of the International Dry Eye WorkShop (2007). The Ocular Surface, 2007; 5(2):163-178.

[16] Herreras J, Pastor JC, Calonge M, Asensio VM.Ocular surface alteration after long-term treatment with an antiglaucomatous drug. Ophthalmology, 1992; 99(7): 1082.

[17] Ishibashi T, Yokoi N, Kinoshita S. Comparison of the short-term effects on the human corneal surface of topical timolol maleate with and without benzalkonium chloride. Journal of Glaucoma, 2003;12(6):486.

[18] Pisella PJ, Debbasch C, Hamard P, Creuzot-Garcher C, Rat P, Brignole F, Baudouin C. Conjunctival proinflammatory and proapoptotic effects of latanoprost and preserved and unpreserved timolol: an ex vivo and in vitro study. Investigative Ophthalmology \& Visual Science, 2004;45(5):1360-68.

[19] de Jong C, Stolwijk T, Kuppens E, de Keizer R, van Best J. Topical timolol with and without benzalkonium chloride: epithelial permeability and autofluorescence of the cornea in glaucoma.

Graefe's Archive for Clinical and Experimental Ophthalmology, 1994;232(4):221-4.

[20] Pauly A, Brignole-Baudouin F, Guenoun JM, Riancho L, Rat P, Warnet JM, Baudouin C.

Comparative study of topical anti-allergic eye drops on human conjunctiva-derived cells: responses 
to histamine and IFN $\gamma$ and toxicological profiles. Graefe's Archive for Clinical and Experimental Ophthalmology, 2007;245(4):534-46.

[21] Craig JP, Purslow C, Murphy PJ, Wolffsohn JS. Effect of a liposomal spray on the pre-ocular tear film. Contact Lens and Anterior Eye, 2010;33(2):83-87.

[22] Yokoi N, Yamada, Mizukusa Y, Bron AJ, Tiffany JM, Kato T, Kinoshita S. Rheology of Tear Film Lipid Layer Spread in Normal and Aqueous Tear-Deficient Dry Eyes. Investigative Ophthalmology \& Visual Science, 2008;49(12):5319-24.

[23] McGinnigle S, Naroo SA, Eperjesi F, Evaluation of Dry Eye. Survey of Ophthalmology, 2012; 57(4):293-316.

[24] Li KY, Yoon G. Changes in aberrations and retinal image quality due to tear film dynamics. Opt. Express, 2006;14(25):12552-12559.

[25] Montés-Micó R A, Cáliz A, Alió JL. Changes in ocular aberrations after instillation of artificial tears in dry-eye patients. Journal of Cataract \& Refractive Surgery, 2004;30(8):1649-52.

[26] Chalmers RL, Begley CL, Caffery B. Validation of the 5-Item Dry Eye Questionnaire (DEQ-5):

Discrimination across self-assessed severity and aqueous tear deficient dry eye diagnoses. Contact Lens and Anterior Eye, 2010;33(2):55-60.

[27] Baudouin C,Labbe A, Liang H, Pauly A, Brignole-Baudouin F. Preservatives in eyedrops: the good, the bad and the ugly. Progress in retinal and eye research, 2010;29(4):312-34.

[28] Thai LC, Tomlinson A, and Ridder III WH, Contact lens drying and visual performance: the vision cycle with contact lenses. Optometry \& Vision Science, 2002;79(6):381-88.

[29] Buscemi P. Retinoscope double pass aberrometry: principles and application of the Nidek OPDScan. In: Krueger RR, Applegate RA, MacRae SM, eds. Wavefront Customized Visual Correction: The Quest for Super Vision II. Thorofare, NJ: SLACK Incorporated; 2004:149-153.

[30] Salmon TO, van de Pol C, Normal-eye Zernike coefficients and root-mean-square wavefront errors. Journal of Cataract \& Refractive Surgery, 2006. 32(12): p. 2064-2074.

[31] Iskander DR, Collins MJ, Davis B, Carney LG. Monochromatic aberrations and characteristics of retinal image quality. Clinical and Experimental Optometry, 2000; 83(6):315-22.

[32] Faul F, Erdfelder E, Lang AG, Buchner A. G* Power 3: A flexible statistical power analysis program for the social, behavioral, and biomedical sciences. Behavior research methods, 2007; 39(2):175-191.

[33] Ridder III WH, LaMotte J, Hall Jr JQ , Sinn R, Nguyen AL, Abufarie L. Contrast sensitivity and tear layer aberrometry in dry eye patients. Optometry \& Vision Science, 2009;86(9):E1059-68.

[34] Tung Cl, Kottaiyan R, Koh S, Wang Q, Yoon , Zavislan M, Davio SR, Aquavella JV. Noninvasive, Objective, Multimodal Tear Dynamics Evaluation of 5 Over-the-Counter Tear Drops in a Randomized Controlled Trial. Cornea, 2012;31(2):108-114.

[35] Berger JS, Head KR, Salmon TO. Comparison of two artificial tear formulations using aberrometry. Clinical and Experimental Optometry, 2009;92(3):206-11.

[36] Liang J, Williams DR. Aberrations and retinal image quality of the normal human eye. Journal of the Optical Society of America. A, Optics image science and vision, 1997;14(11):2873-83.

[37] Rozema JJ, Van Dyck DE, and Tassignon MJ. Clinical comparison of 6 aberrometers Part 2: Statistical comparison in a test group. Journal of Cataract \& Refractive Surgery, 2006;32(1):33-44.

[38] Atchison DA, Collins MJ, Wildsoet CF, Christensen J, Waterworth MD. Measurement of monochromatic ocular aberrations of human eyes as a function of accommodation by the Howland aberroscope technique. Vision Research, 1995;35(3):313-23.

[39] He JC, Marcos S, webb RH, Burns SA, Measurement of the wave-front aberration of the eye by a fast psychophysical procedure. Journal of the Optical Society of America. A, Optics image science and vision.1998. 15(9):2449-56.

[40] Rodríguez Pérez, PNavarro R, Arines J, Bara S. A calibration set for ocular aberrometers: Manufacture, testing and application. Digital CSIC, 2006. http://hdl.handle.net/10261/35625. 


\begin{tabular}{cccccc}
\multicolumn{2}{c}{ Treatment } & $\begin{array}{c}\text { Unpreserved hypromellose } \\
\text { drops }\end{array}$ & $\begin{array}{c}\text { Unpreserved liposome } \\
\text { spray }\end{array}$ & Combination & $\begin{array}{c}\text { No } \\
\text { preference }\end{array}$ \\
\hline \multirow{2}{*}{ Subjects } & Normal & $66.60 \%$ & $33.40 \%$ & 0 & 0 \\
& Dry & $37.60 \%$ & $50 \%$ & $8.40 \%$ & $4 \%$
\end{tabular}

Table 1

\begin{tabular}{|c|c|c|c|c|c|c|c|c|c|}
\hline & \multicolumn{3}{|c|}{ Baseline visit Hypromellose Drops } & \multicolumn{3}{|c|}{ Baseline visit Liposome Spray } & \multicolumn{3}{|c|}{ Baseline Visit Combination } \\
\hline & $\begin{array}{c}\text { Normal } \\
\text { Eyes }\end{array}$ & Dry eyes & $\begin{array}{c}P \\
\text { value }\end{array}$ & Normal Eyes & Dry Eyes & $\begin{array}{l}P \\
\text { value }\end{array}$ & Normal Eyes & Dry Eyes & $\begin{array}{c}P \\
\text { value }\end{array}$ \\
\hline & $0.105 \pm$ & $0.100 \pm$ & & $0.085 \pm$ & $0.106 \pm$ & & $0.090 \pm$ & $0.104 \pm$ & \\
\hline \multirow[t]{2}{*}{ Coma $(\mu \mathrm{m})$} & 0.045 & 0.050 & 0.75 & 0.042 & 0.048 & 0.12 & 0.056 & 0.057 & 0.37 \\
\hline & $0.044 \pm$ & $0.036 \pm$ & & $0.041 \pm$ & $0.039 \pm$ & & $0.040 \pm$ & $0.058 \pm$ & \\
\hline Spherical aberrations $(\mu \mathrm{m})$ & 0.033 & 0.022 & 0.34 & 0.029 & 0.029 & 0.87 & 0.027 & 0.078 & 0.36 \\
\hline Total higher order & $0.218 \pm$ & $0.237 \pm$ & & $0.204 \pm$ & $0.238 \pm$ & & $0.213 \pm$ & $0.239 \pm$ & \\
\hline aberrations $(\mu \mathrm{m})$ & 0.078 & 0.078 & 0.29 & 0.075 & 0.063 & 0.14 & 0.091 & 0.084 & 0.21 \\
\hline
\end{tabular}

Table 2

\begin{tabular}{|c|c|c|c|c|c|c|c|c|c|c|c|c|}
\hline & \multicolumn{4}{|c|}{ Hypromellose drops } & \multicolumn{4}{|c|}{ Liposome spray } & \multicolumn{4}{|c|}{ Hypromellose drops and liposome spray } \\
\hline & \multicolumn{2}{|c|}{ Normal Eyes } & \multicolumn{2}{|c|}{ Dry eyes } & \multicolumn{2}{|c|}{ Normal Eyes } & \multicolumn{2}{|c|}{ Dry eyes } & \multicolumn{2}{|c|}{ Normal Eyes } & \multicolumn{2}{|c|}{ Dry eyes } \\
\hline & Baseline & After 1 hour & Baseline & After 1 hour & Baseline & After 1 hour & Baseline & After 1 hour & Baseline & After 1 hour & Baseline & After 1 hour \\
\hline & $0.105 \pm$ & $0.119 \pm$ & $0.101 \pm$ & $0.107 \pm$ & $0.108 \pm$ & $0.106 \pm$ & $0.107 \pm$ & $0.103 \pm$ & $0.091 \pm$ & $0.089 \pm$ & $0.104 \pm$ & $0.109 \pm$ \\
\hline \multirow[t]{2}{*}{ Coma $(\mu \mathrm{m})$} & 0.045 & 0.115 & 0.050 & 0.043 & 0.047 & 0.053 & 0.048 & 0.047 & 0.057 & 0.049 & 0.058 & 0.047 \\
\hline & $0.044 \pm$ & $0.040 \pm$ & $0.036 \pm$ & $0.035 \pm$ & $0.041 \pm$ & $0.038 \pm$ & $0.040 \pm$ & $0.035 \pm$ & $0.040 \pm$ & $0.040 \pm$ & $0.058 \pm$ & $0.038 \pm$ \\
\hline \multirow[t]{2}{*}{ Spherical aberrations $(\mu \mathrm{m})$} & 0.030 & 0.029 & 0.022 & 0.021 & 0.029 & 0.029 & 0.029 & 0.019 & 0.027 & 0.029 & 0.079 & 0.023 \\
\hline & $0.218 \pm$ & $0.213 \pm$ & $0.237 \pm$ & $0.243 \pm$ & $0.204 \pm$ & $0.209 \pm$ & $0.238 \pm$ & $0.243 \pm$ & $0.213 \pm$ & $0.221 \pm$ & $0.239 \pm$ & $0.247 \pm$ \\
\hline Total higher order aberrations $(\mu \mathrm{m})$ & 0.079 & 0.075 & 0.078 & 0.062 & 0.075 & 0.082 & 0.063 & 0.073 & 0.091 & 0.084 & 0.084 & 0.084 \\
\hline
\end{tabular}




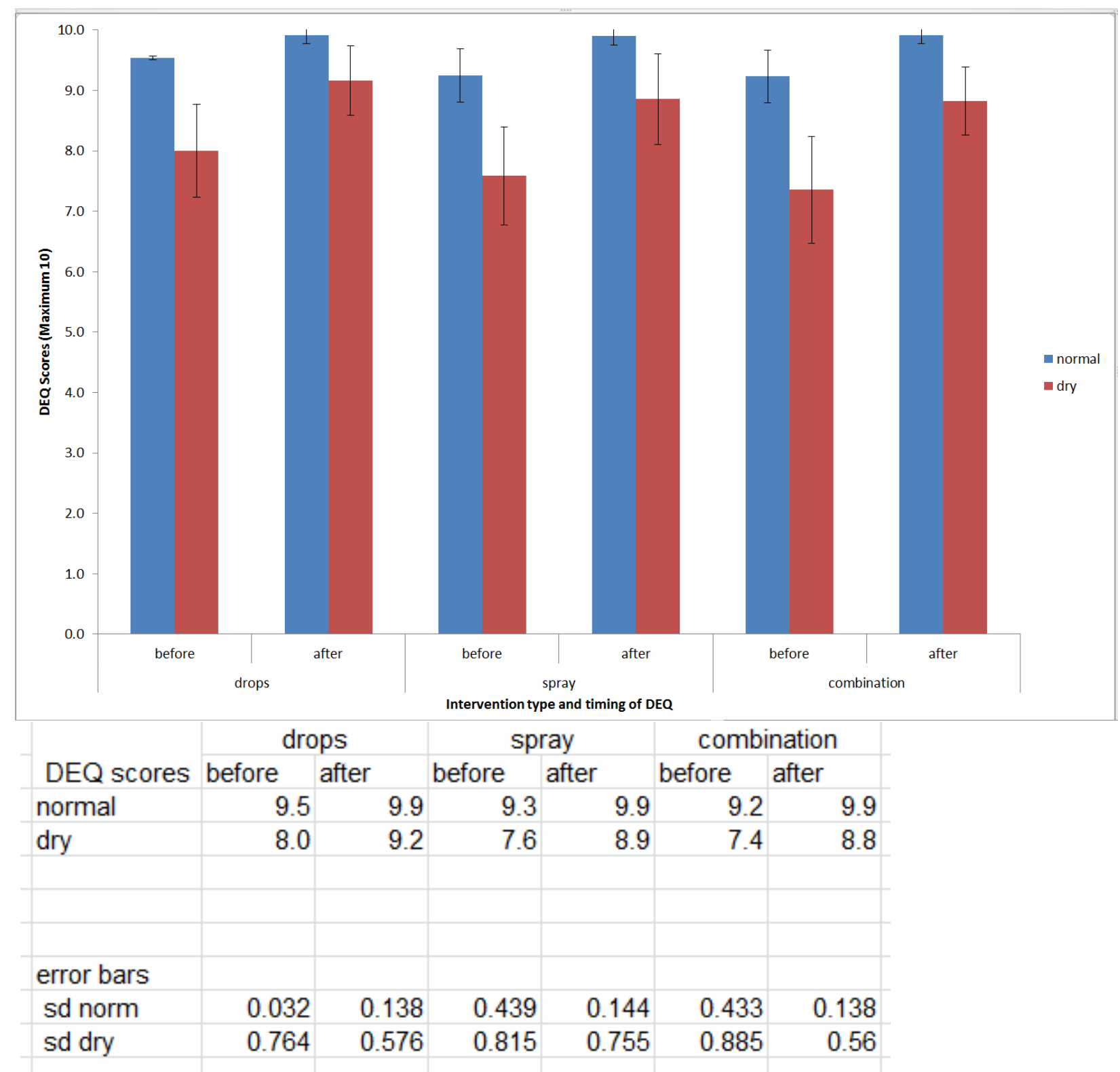

Fig. 1. Comfort scores out of 10 , before and one hour after treatment $(n=24$ for each group), where 10 represents the most comfortable (SD indicated).

Table 1. Treatment preferences for all subjects ( $n=24$ for each group)

Table 2. Results of independent t-test for baseline total higher order aberrations between normal $(n=24)$ and dry $(n=24)$ eyes on each separate visit

Table 3. Mean and standard deviation for higher order aberrations in normal $(n=24)$ and dry $(n=24)$ groups for each intervention at baseline and when measured one hour later. 